\title{
The Isolation and Properties of the Yeast Cell Vacuole
}

\author{
By K. J. INDGE \\ Department of Biochemistry, University of Manchester Institute of \\ Science and Technology, Manchester
}

(Accepted for publication 26 October 1967)

SUMMARY

When glucose and a chelating agent such as EDTA were added to yeast protoplasts suspended in $8.5 \%(\mathrm{w} / \mathrm{v})$ mannitol solution at $\mathrm{pH} 6.4$ almost all the protoplasts lysed and the main vacuole was released as a discrete structure from about $75 \%$ of the protoplasts. The isolated vacuoles were themselves sensitive to osmotic shock and exhibited vital-staining reactions. They are therefore considered to be relatively undamaged. Some of the properties of the isolated vacuoles are described.

\section{INTRODUCTION}

Since the development of a method for isolating protoplasts from yeast cells (Eddy \& Williamson, 1957) these bodies have become a favoured starting material for the isolation of various subcellular components. Eddy (1959) observed that ultrasonic vibration in certain circumstances released the main vacuole from the protoplast in a relatively intact form, but he did not develop a reliable method for preparing these bodies. Other methods of fragmenting the protoplasts include lysis in hypotonic media (Duell, Inoue \& Utter, I964; Boulton, 1965), ultraviolet irradiation (Svihla, Schlenk \& Dainko, I96I) and treatment with detergent (Rozijn \& Tonino, 1964). Such procedures have been used to prepare mitochondria, cytoplasmic membranes, vacuoles and nuclei respectively. Following the investigation into the stability of resting and metabolizing yeast protoplasts (preceding papers) it became apparent that the phenomenon of metabolic lysis (Abrams, 1959, I960) might provide the basis of an alternative method of preparing subcellular structures. This is illustrated in the present work in which apparently intact vacuoles have been prepared from yeast protoplasts lysed metabolically in the presence of EDTA and glucose.

\section{METHODS}

Yeast protoplasts. Stock suspensions of protoplasts (about $\mathrm{IO}^{9} / \mathrm{ml}$.) of the yeast Saccharomyces carlsbergensis NCYC 74 were prepared as described previously (Indge, I968). Protoplasts stored at $0^{\circ}$ for 3 days and then washed exhibited the same behaviour during metabolic lysis as freshly prepared protoplasts.

Metabolic lysis. Samples (I vol.) of the stock protoplast suspension were added to 50-IOO vol. of $0.0 \mathrm{I}$ M-imidazole $\mathrm{HCl}$ buffer $(\mathrm{pH} \mathrm{6.4)}$ containing glucose $(0.5 \%$, w/v) and selected concentrations of mannitol, citrate ions or EDTA. Citrate ion and EDTA were added as the tris salts. The mixture was incubated at $30^{\circ}$ for up to $20 \mathrm{~min}$. and the progress of the metabolic lysis reaction was followed by measurements of extinction in the Hilger Spekker using neutral filters (Ilford H. 508) and I cm. cells. 
Application of vital stains. Since contact with neutral red and toluidine blue was found to lyse both the protoplasts and the isolated vacuoles the behaviour of these bodies with the dyes was observed by irrigating slide preparations with isotonic solutions of the dyes. The dye concentrations given are those used in the irrigation fluid. By following the movement of the dye front across the slide it was possible to obtain photographs of the stained bodies before lysis occurred.

\section{RESULTS}

\section{Metabolic lysis}

It was shown in the preceding paper (Indge, 1968) that when yeast protoplasts $\left(2 \times 10^{7} / \mathrm{ml}\right.$.) were incubated at $30^{\circ}$ in a solution containing mannitol $(10 \%, \mathrm{w} / \mathrm{v})$, citrate ions $(6 \mathrm{mM})$ or EDTA $(5 \mathrm{~mm})$ and glucose $(0.5 \%, \mathrm{w} / \mathrm{v})$ at $\mathrm{pH} 6.4$ rapid metabolic lysis ensued. Examination by phase-contrast microscope of suspensions of protoplasts which were undergoing metabolic lysis revealed that the protoplasts swelled progressively and finally burst. As they lysed the protoplasts released spherical bodies 2-3 $\mu$ in diameter, each protoplast giving one such body, on average. These bodies have been identified with the cell vacuole. When the various particles released were collected by centrifugation at $2000 \mathrm{~g}$ for $4 \mathrm{~min}$., the pellet consisted of a gelatinous membraneous material, vacuoles and unlysed protoplasts.

\section{Conditions of metabolic lysis}

By using the conditions described above, up to $25 \%$ of the protoplasts remained intact after incubation for $30 \mathrm{~min}$. The composition of the lysis medium was therefore varied with the aim of decreasing the amount of this fraction. Figure I shows the effect of varying the concentration of mannitol in the lysis medium on the extinction changes taking place during lysis. Previous work had shown that the extinction as determined in these circumstances was a linear function of the number of unburst protoplasts (Indge, I968). It can be seen that both the initial rate of change and the overall change in extinction increased as the concentration of mannitol in the medium approached $8 \%(\mathrm{w} / \mathrm{v})$. Some of the protoplasts in the $8 \%(\mathrm{w} / \mathrm{v})$ mannitol solution burst even in the absence of glucose (Fig. I) and released intact vacuoles. On the basis of further similar experiments it was established that high yields of vacuoles, associated with extensive lysis of the protoplast population in the presence of glucose, occurred when the mannitol concentration was $8.5 \%(\mathrm{w} / \mathrm{v})$.

A second factor which influenced lysis was the suspension density of the protoplasts in the test medium. Metabolic lysis was done with suspension densities of I, 5 and 10 times that used in the above experiments $\left(2 \times 10^{7} / \mathrm{ml}\right.$.), the suspensions being diluted appropriately for the purpose of measuring their extinctions. As shown in Fig. 2, although the initial rates of lysis in the three tests were similar, the number of protoplasts which lysed was smaller at the higher suspension densities. Moreover, the vacuoles and other membraneous elements of the protoplasts which lysed in the high density suspensions tended to remain attached to one another, forming structures which retained the gross morphology of the parent protoplasts. These results suggested that during lysis the protoplasts liberated some factor into the medium, possibly $\mathrm{K}^{+}$or $\mathrm{Mg}^{2+}$, which stabilized the unlysed protoplasts. On the basis of the above results a solution containing mannitol $(8.5 \%, \mathrm{w} / \mathrm{v})$, glucose $0.5 \%(\mathrm{w} / \mathrm{v})$, 0.0I M-imida- 
zole $\mathrm{HCl}$ buffer ( $\mathrm{pH} 6 \cdot 4)$ and citrate ions $(6 \mathrm{mM})$ or EDTA ( $5 \mathrm{mM})$ was expected to give the best yield of vacuoles. The initial suspension density of the protoplasts was about $2 \times 10^{7} / \mathrm{ml}$. corresponding to an extinction of $0.5-0.6(\mathrm{I} \mathrm{cm}$. cells). By using these conditions, between 95 and $100 \%$ of the protoplasts lysed during 20 min. at $30^{\circ}$; at least $75 \%$ of the lysed protoplasts gave vacuoles.

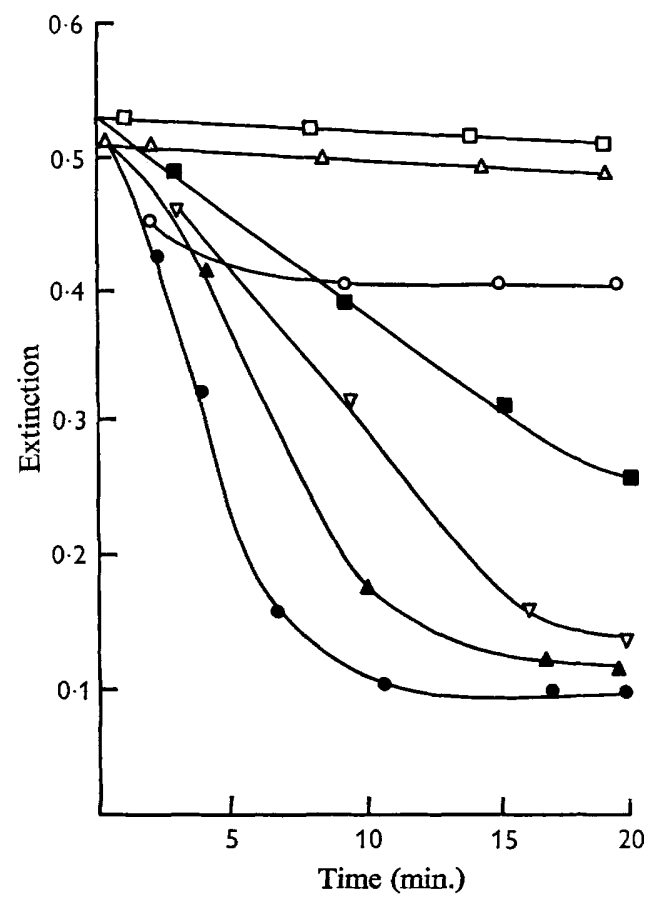

Fig. I

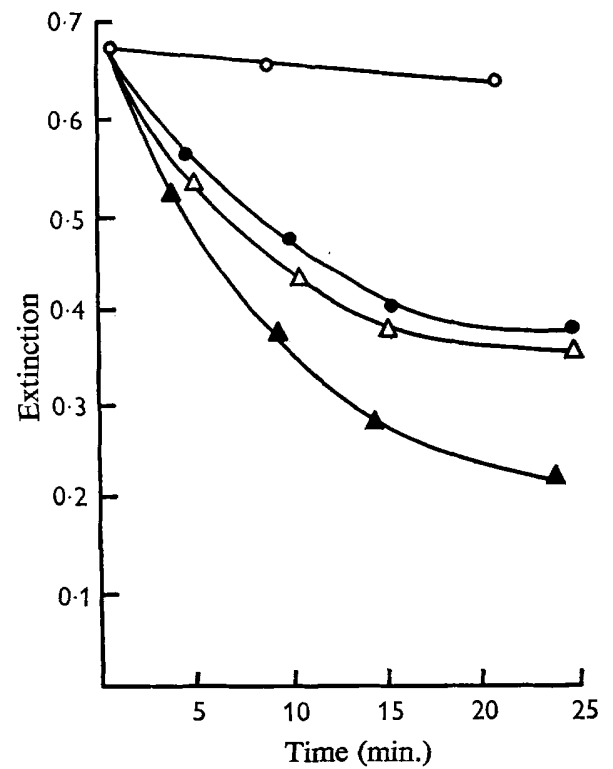

Fig. 2

Fig. I. The effect of the concentration of mannitol in the medium on metabolic lysis of yeast protoplasts. Protoplasts were incubated at $30^{\circ}$ in solutions containing: $0.01 \mathrm{M}$-imidazole $\mathrm{HCl}$ buffer (pH 6.4); glucose $(0.5 \%$, w/v); citrate $(6 \mathrm{mM})$; mannitol $(\%, w / v)$ I 5 ( I $2(\nabla-\nabla)$, Io $(\Delta-\triangle)$ or $8(--))$. Control tests containing no glucose with mannitol $(\%, w / v)$ is $(\square-\square)$, Io $(\triangle-\triangle), 8(\mathrm{O}-\mathrm{O})$.

Fig. 2. The influence of the suspension concentration of yeast protoplasts on metabolic lysis. Protoplasts suspended in: $0.01 \mathrm{M}$-imidazole $\mathrm{HCl}$ buffer $(\mathrm{pH} 6.4)$; glucose $(0.5 \%$, w/v); citrate

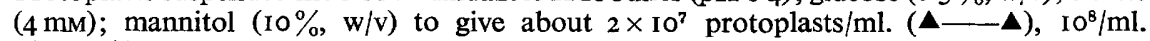
$(\triangle-\triangle), 2 \times 10^{8} / \mathrm{ml}$. (- Tests containing $10^{8}$ and $2 \times 10^{9}$ protoplasts $/ \mathrm{ml}$. were diluted before reading their extinctions. Control tests without glucose and $2 \times 10^{7}$ protoplasts $/ \mathrm{ml}$. $(\mathrm{O}-\mathrm{O})$.

The liberated vacuoles were concentrated by centrifugation at $2000 \mathrm{~g}$ for $4 \mathrm{~min}$., when the vacuoles tended to remain dispersed in the pellet which formed at the bottom of the centrifuge tube, whereas unlysed protoplasts and other particles collected as a gelatinous, macroscopic aggregate. When the pellet was resuspended in a solution containing mannitol (10\%, w/v) and $0.0 \mathrm{I} \mathrm{M}$-imidazole $\mathrm{HCl}$ buffer $(\mathrm{pH} \mathrm{6.4})$ the gelatinous residue did not disperse and readily sedimented under gravity, leaving a supernatant solution rich in vacuoles which could be decanted from the other constituents. The appearance in the light microscope (P1. I, fig. I) and the electron microscope (Pl. 3, fig. 7) of the material so obtained is shown. 


\section{Identification of the vacuole}

A property common to many vacuoles as they occur in vivo is their ability to accumulate certain dyes (Zirkle, 1937). This so-called vital-staining reaction has been studied in the yeast cell by Guilliermond (I94I). It seemed important to know whether the vacuole bodies released from the protoplasts during metabolic lysis would stain in a similar fashion.

The uptake of neutral red (0.0 \% $\%$ w/v) at $\mathrm{pH} 7.0$ by resting yeast cells followed the pattern described by Guilliermond (I94I). Initially many stained granules exhibiting Brownian movement were observed in the vacuole; these became deposited against the vacuole membrane (Pl. I, fig. 2) and coalesced to form large red globules which slowly dissolved in the vacuolar sap (Pl. I, fig. 3). At $\mathrm{pH} 6.4$ yeast protoplasts were stained similarly by neutral red (Pl. 2, fig. 5). A preparation of the bodies liberated by metabolic lysis of the protoplasts and stained with $0.005 \%(\mathrm{w} / \mathrm{v})$ neutral red is shown in P1. 2, fig. 4. The dye caused the bodies to clump to some extent, and as staining continued, lysis ensued, apparently with the ejection of 'fingers' of precipitated dye which then rapidly dissolved. A few strongly stained particles were also released during lysis of the vacuoles.

The similarities between the interaction of these bodies with neutral red and the in vivo staining of the cell vacuole indicates that they are derived from the cell vacuole and are relatively undamaged. A similar parallelism existed between the reactions of the vacuoles in vivo and in vitro with toluidine blue $(0.05 \%, \mathrm{w} / \mathrm{v})$. Isolated vacuoles were also lysed by this dye, with the liberation of large numbers of stained particles and a few rod-like structures (Townsend \& Lindegren, 1953).

\section{Physical properties of the isolated vacuoles}

Fresh preparations of vacuoles adhered strongly to glass slides; this property was lost when the vacuoles were stored for $4-8 \mathrm{hr}$ at $0^{\circ}$. Once the vacuoles were attached to a slide they could be drawn out to form beaded threads or long filaments, often without loss of optical contrast, by flooding isotonic buffer solution across the slide. Solutions flowing at low shear rates distorted the vacuoles, which reverted to their original shape, however, when the stress was removed. These circumstances made it possible to investigate the effects of certain compounds on selected groups of vacuoles by irrigating test solutions across the slide.

The isolated vacuoles were osmotically active in that they reversibly swelled and contracted as the concentration of mannitol in the medium was varied between $7 \cdot 5$ and I I \% (w/v). At higher concentrations of mannitol excessive contraction occurred with the formation of membrane 'tails' around the vacuoles. This process was not reversed when the osmotic pressure of the medium was decreased and the vacuoles lysed. The vacuoles lysed in $5-6 \%(\mathrm{w} / \mathrm{v})$ mannitol solution. During this lysis the optical contrast of the vacuole rapidly faded, leaving a spherical membrane ghost with no visible fracture in its surface. The ghosts remained adsorbed to the slide.

\section{Stability of vacuoles}

The stability of isolated vacuoles was investigated with the phase-contrast microscope. Isolated vacuoles survived $\mathrm{I}-2$ days at $0^{\circ}$ when stored as a thick suspension in 
mannitol solution $(10 \%, \mathrm{w} / \mathrm{v})$ at $\mathrm{pH} 6.4$; at $30^{\circ}$ considerable lysis took place within $30 \mathrm{~min}$.

In an attempt to stabilize preparations of isolated vacuoles certain compounds were added to the buffered mannitol $(10 \%, w / v)$ solution in which they were routinely suspended. The compounds tested included the chlorides, phosphates and sulphates of $\mathrm{Na}^{+}, \mathrm{K}^{+}, \mathrm{Mg}^{2+}$ and $\mathrm{Ca}^{2+}(0.005-0.05 \mathrm{M})$ which are known to stabilize plant cell vacuoles (Eichberger, I933), and bovine serum albumin ( $1 \%, \mathrm{w} / \mathrm{v})$, dextran 500 (Pharmacia, 2\%, w/v), dextran 40 (Pharmacia, 2\%, w/v) and Ficoll (Pharmacia, $\mathrm{I}-5 \%, \mathrm{~W} / \mathrm{v})$. None of these compounds appeared to inhibit the spontaneous lysis of the vacuoles at $30^{\circ}$. Magnesium chloride $\left(\mathrm{O}^{\circ} \mathrm{OI} \mathrm{M}\right)$ and calcium chloride (O.OI M) precipitated the contents of the vacuoles, giving spheres which exhibited Brownian movement (Pl. 2, fig. 6). The presence of these ions also caused the vacuoles to aggregate.

\section{DISCUSSION}

The success of the present technique in liberating apparently intact vacuoles from yeast protoplasts results from the change in the permeability properties of the protoplast membrane induced by glucose and chelating agents. Under the conditions of the lysis experiments the osmotic stabilizer, mannitol, is no longer excluded from the protoplast and lysis results. The survival of the various cellular organelles is then influenced directly by the composition of the medium. Since the vacuole survives the lysis reaction it may be inferred that the vacuole membrane is relatively impermeable to mannitol under these conditions and that its properties differ from those of the protoplast membrane. Other investigators have reported differences between the properties of the protoplast and vacuolar membranes (Bartholomew \& Mittwer, 1952; Svihla, Dainko \& Schlenk, 1963).

The lysis conditions used in this work allow the preservation of the cell vacuole; unpublished observations in these laboratories have shown that nuclei prepared according to Rozijn \& Tonino (1964) were unstable when suspended in the lysis medium. The fate of other subcellular structures, such as mitochondria and the endoplasmic reticulum, during metabolic lysis is unknown. It is possible that other subcellular organelles might be prepared by using the metabolic lysis technique with suitable control of the composition of the medium. The possibility that vacuoles might serve as a model system for the study of transport phenomena was entertained at an early stage in this work. The failure to find conditions which decreased the lysis of the isolated vacuoles has so far prevented work in this direction.

Since the completion of this work Matile \& Wiemken (I967) have published an alternative method for the liberation and purification of vacuoles from yeast. Vacuoles prepared by their method are smaller and show a larger variation in size $\left(0 \cdot 2-I^{\circ} 0 \mu\right.$ diameter) than do vacuoles prepared by metabolic lysis (2-3 $\mu$ diam.). The reason for these differences is not clear.

The author wishes to thank Professor A. A. Eddy for his interest in this work and for his suggestions concerning the manuscript. The author is also grateful to Miss $\mathrm{C}$. Backhouse for preparing the electron micrograph. 


\section{REFERENCES}

Abrams, A. (1959). Reversible metabolic swelling of bacterial protoplasts. J. biol. Chem. 234, 383.

ABRAMS, A. (1960). Metabolically dependent penetration of oligosaccharides into bacterial cells and protoplasts. J. biol. Chem. 235, I281.

Bartholomew, J. W. \& MrtTwer, T. (1952). Cellular structure as revealed by ultraviolet photolysis and the electron microscope. J. Bact. 64, I.

Boulton, A. A. (1965). Some observations on the chemistry and morphology of the membranes released from yeast protoplasts by osmotic shock. Exp. Cell Res. 37, 343.

Duell, E. A., Inoue, S. \& UTTER, M. F. (I964). Isolation and properties of intact mitochondria from spheroplasts of yeast. J. Bact. 88, 1762 .

EDDY, A. A. (1959). The probable nuclear origin of certain of the bodies released from yeast protoplasts by ultrasonic treatment. Exp. Cell Res. 17, 447.

Eddy, A. A. \& Williamson, D. H. (1957). A method of isolating protoplasts from yeast. Nature, Lond. 179, 1252.

Eichberger, R. (1933). Über die 'lebensdauer' isolierter Tonoplasten. Protoplasma 20, 606.

Guilliermond, A. (I94I). In The Cytoplasm of the Plant Cell. Waltham, Mass., U.S.A.: Chronica Botanica Company.

INDGE, K. J. (1968). Metabolic lysis of yeast protoplasts. J. gen. Microbiol. 5I, 433.

Matile, Ph. \& Wiemken, A. (1967). The vacuole as the lysosome of the yeast cell. Arch. Mikrobiol. 56, 148.

RoziJn, Th. H. \& Tonino, G. J. M. (I964). Studies on the yeast nucleus. I. The isolation of nuclei. Biochim. biophys. Acta 91, 105.

Svihla, G., Dainko, J. L. \& SChlenk, F. (1963). Ultraviolet microscopy of purine compounds in the yeast vacuole. J. Bact. 85, 399.

Svihla, G., Schlenk, F. \& DainKo, J. L. (I961). Spheroplasts of the yeast Candida utilis. J. Bact. 82, 808.

TownSEND, G. F. \& LINDEGREN, C. C. (1953). Structures in the yeast cell revealed in wet mounts. Cytologia, 18, 7 .

ZIRKLE, C. (1937). The plant vacuole. Bot. Rev. 3, I.

\section{EXPLANATION OF PLATES}

\section{Plate I}

Fig. I. Yeast vacuoles isolated by metabolic lysis. Phase microscopy. $\times 3500$.

Fig. 2. Yeast cells during staining with neutral red $0.01 \%(w / v)$. Note globules of dye around the vacuole wall. $\times 1900$.

Fig. 3. A later stage in the staining of yeast cells with neutral red $0.01 \%(w / v)$. The dye has begun to dissolve in the vacuole sap. $\times 1700$.

\section{Plate 2}

Fig. 4. Isolated vacuoles stained with neutral red $0.005 \%(\mathrm{w} / \mathrm{v}) . \times 2250$.

Fig. 5. Yeast protoplasts stained with neutral red $0.01 \%(\mathrm{w} / \mathrm{v}) \times 2250$.

Fig. 6. Isolated yeast vacuoles suspended in $10 \%(\mathrm{w} / \mathrm{v})$ mannitol solution containing $0.01 \mathrm{M}-\mathrm{MgCl}_{2}$. The vacuolar sap has separated into a dark sphere contained within the vacuole wall. Phase microscopy. $\times 3000$.

\section{Plate 3}

Fig. 7. Electron micrograph of a crude preparation of yeast vacuoles. The preparation was fixed overnight at $0^{\circ}$ in 0.01 M-imidazole $\mathrm{HCl}$ buffer $(\mathrm{pH} 6.4)+$ mannitol to $10 \%(\mathrm{w} / \mathrm{v})+5 \%(\mathrm{w} / \mathrm{v})$ glutaraldehyde; then stained in $5 \%(\mathrm{w} / \mathrm{v})$ aqueous $\mathrm{KMnO}_{4}$ for $2 \mathrm{hr}$ at $0^{\circ} . \times 14,000$. 
Journal of General Microbiology, Vol. 5I, No. 3

Plate I
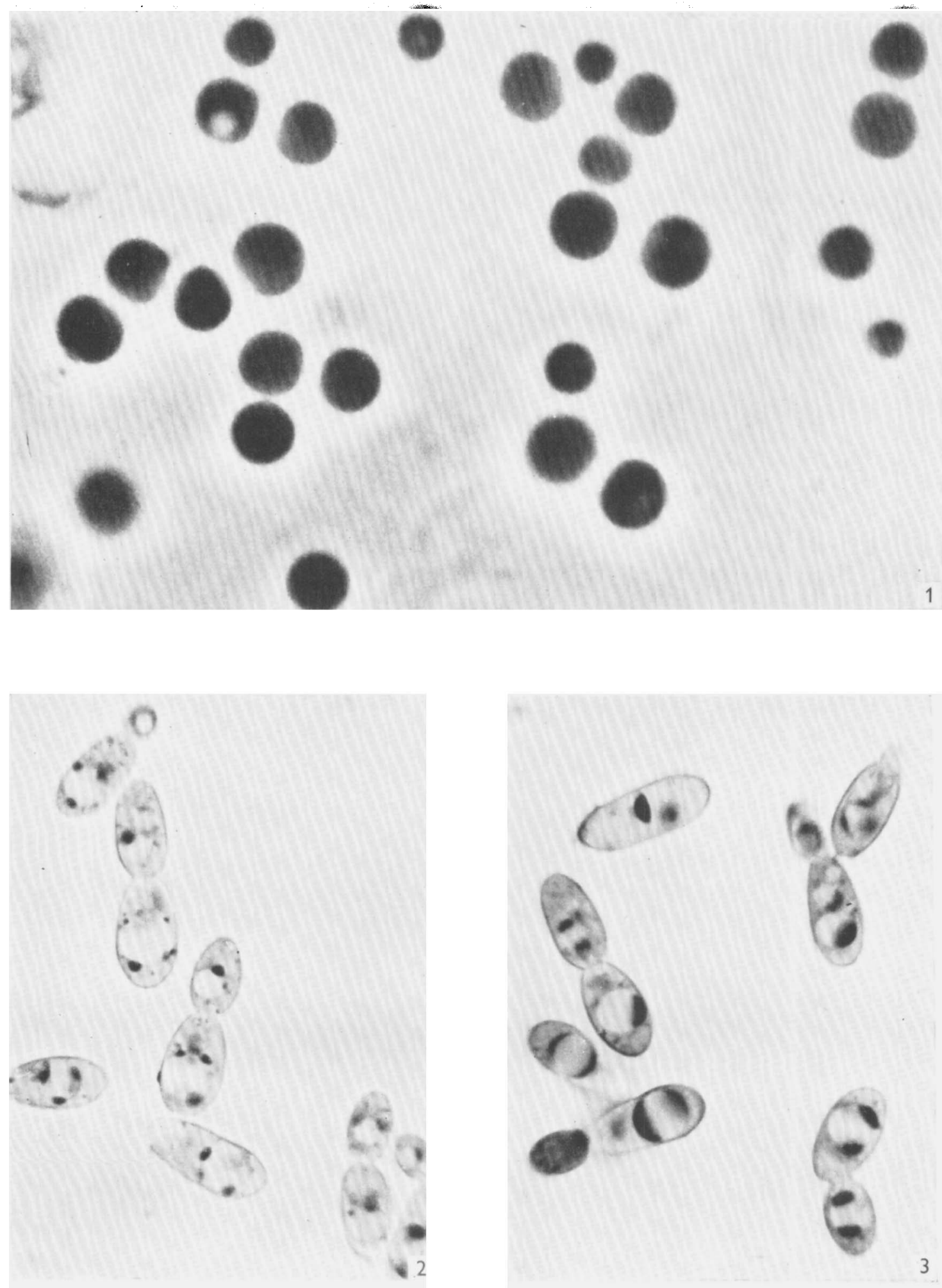
Journal of General Microbiology, Vol. 5I, No. 3

Plate 2
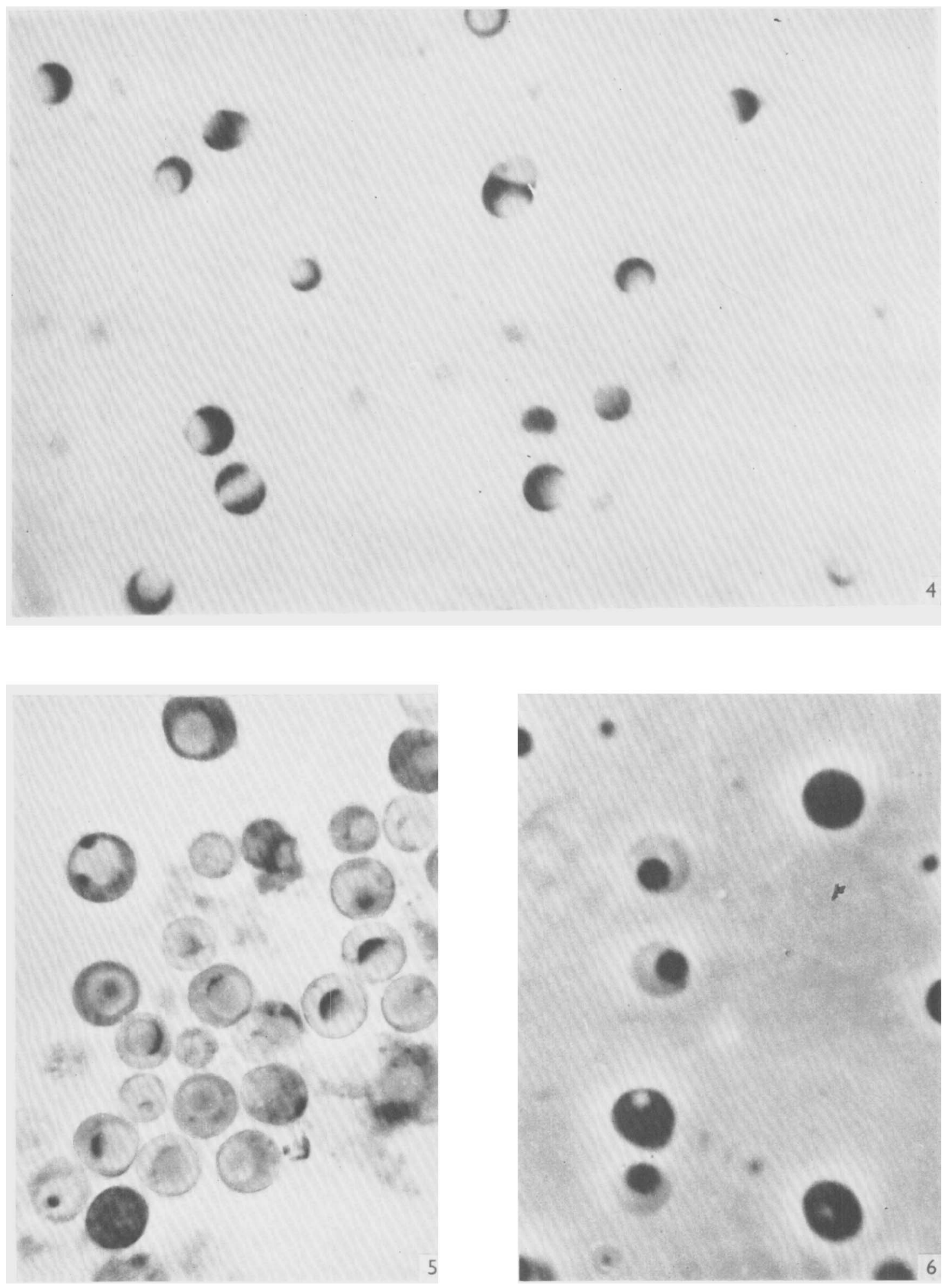

K. J. INDGE 
Journal of General Microbiology, Vol. 5I, No. 3

Plate 3

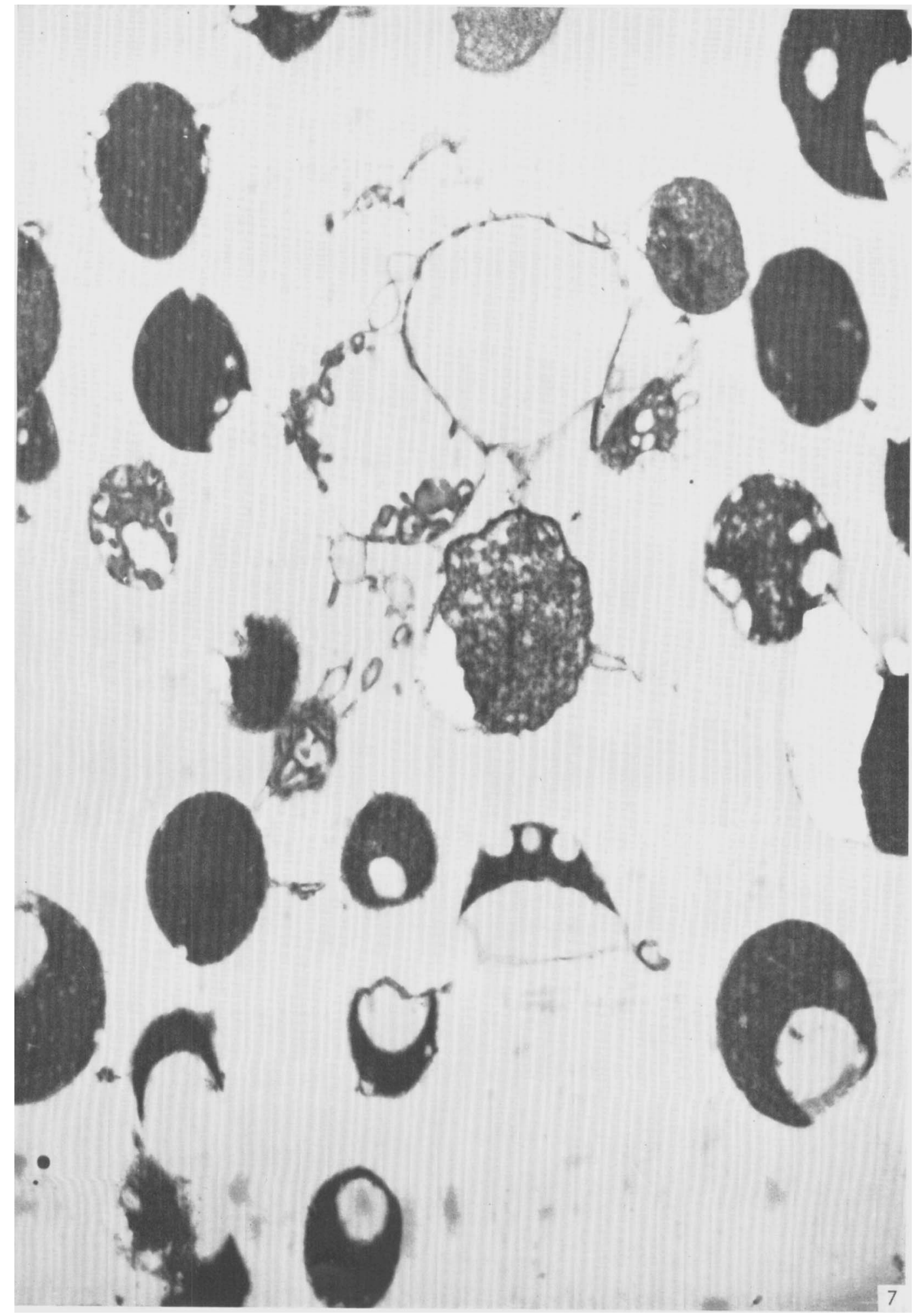

K. J. INDGE 